\title{
Incidental germline findings during molecular profiling of tumor tissues for precision oncology: molecular survey and methodological obstacles
}

\author{
Alexandra Lebedeva ${ }^{1,2^{*}}$ (D) Yulia Shaykhutdinova ${ }^{1}$, Daria Seriak ${ }^{3}$, Ekaterina Ignatova ${ }^{1,4,5}$, Ekaterina Rozhavskaya ${ }^{1}$, \\ Divyasphoorthi Vardhan ${ }^{6}$, Sofia Manicka ${ }^{6}$, Margarita Sharova ${ }^{1}$, Tatiana Grigoreva' ${ }^{1}$, Ancha Baranova ${ }^{6}$, \\ Vladislav Mileyko ${ }^{1}$ and Maxim Ivanov ${ }^{1,7}$
}

\begin{abstract}
Background: A fraction of patients referred for complex molecular profiling of biopsied tumors may harbor germline variants in genes associated with the development of hereditary cancer syndromes (HCS). Neither the bioinformatic analysis nor the reporting of such incidental germline findings are standardized.

Methods: Data from Next-Generation Sequencing (NGS) of biopsied tumor samples referred for complex molecular profiling were analyzed for germline variants in HCS-associated genes. Analysis of variant origin was performed employing bioinformatic algorithms followed by manual curation. When possible, the origin of the variant was validated by Sanger sequencing of the sample of normal tissue. The variants' pathogenicity was assessed according to ACMG/AMP.
\end{abstract}

Results: Tumors were sampled from 183 patients (Males: 75 [41.0\%]; Females: 108 [59.0\%]; mean [SD] age, 57.7 [13.3] years) and analysed by targeted NGS. The most common tumor types were colorectal (19\%), pancreatic (13\%), and lung cancer (10\%). A total of 56 sequence variants in genes associated with HCS were detected in 40 patients. Of them, 17 variants found in 14 patients were predicted to be of germline origin, with 6 variants interpreted as pathogenic (PV) or likely pathogenic (LPV), and 9 as variants of uncertain significance (VUS). For the 41 out of 42 (97\%) missense variants in HCS-associated genes, the results of computational prediction of variant origin were concordant with that of experimental examination. We estimate that Sanger sequencing of a sample of normal tissue would be required for $\sim 1-7 \%$ of the total assessed cases with PV or LPV, when necessity to follow with genetic counselling referral in $\sim 2-15 \%$ of total assessed cases (PV, LPV or VUS found in HCS genes).

Conclusion: Incidental findings of pathogenic germline variants are common in data from cancer patients referred for complex molecular profiling. We propose an algorithm for the management of patients with newly detected variants in genes associated with HCS.

*Correspondence: lebedeva@oncoatlas.ru

${ }^{1}$ Atlas Oncodiagnostics, LLC, Moscow, Russia

Full list of author information is available at the end of the article

\section{Introduction}

NGS is gaining recognition as an in vitro companion diagnostic aid in clinical decision-making. In 2017, Oncomine DX Target Test became the first NGS-based test approved by the FDA for a set of non-small-cell lung permits use, sharing, adaptation, distribution and reproduction in any medium or format, as long as you give appropriate credit to the original author(s) and the source, provide a link to the Creative Commons licence, and indicate if changes were made. The images or other third party material in this article are included in the article's Creative Commons licence, unless indicated otherwise in a credit line to the material. If material is not included in the article's Creative Commons licence and your intended use is not permitted by statutory regulation or exceeds the permitted use, you will need to obtain permission directly from the copyright holder. To view a copy of this licence, visit http://creativecommons.org/licenses/by/4.0/. The Creative Commons Public Domain Dedication waiver (http://creativeco mmons.org/publicdomain/zero/1.0/) applies to the data made available in this article, unless otherwise stated in a credit line to the data. 
cancer-related genetic alterations [1, 2]. This was followed shortly by FoundationOne CDx [3, 4], FoundationFocus CDxBRCA [5], and MyChoice HRD CDx [6]. In the context of managing oncology patients, NGS is predominantly used as a tool for predicting the efficacy of therapies that may be influenced by the presence or lack of specific somatic mutations [7].

Compared to conventional methods for DNA analysis, such as Sanger sequencing or PCR (Polymerase Chain Reaction), NGS can identify a large array of DNA regions, which are not limited to the short list of mutations that clinicians expect to find in a patient with a certain diagnosis. Hence, a typical result of diagnostic NGS is represented by a list of identified mutations, only some of which are related to the specific disease phenotype, and others, unrelated to the specific disease, that have the potential for clinical relevance [8]. The latter type of reported variance is known as "incidental", or secondary findings. Incidental findings of germline origin are especially important for both managing the patients' health and correctly assessing the risks of their relatives developing pathologies $[9,10]$.

The reporting of germline findings usually follows the ACMG/AMP guidelines or their refined version, the SHERLOC guidelines. These guidelines propose that each sequence variant should be assessed according to a 5-Tier system based on objective criteria, such as population frequency of a genetic alteration, computational predictions of pathogenicity, or existing research on the functional effect of a genetic variant. However, existing guidelines are not disease-specific, and are mostly suitable for hereditary diseases associated with highly penetrant genes. Next, the criteria in the ACMG/AMP or SHERLOC guidelines do not include the medical history of the proband in the decision-making process. Another set of existing guidelines, NCCN, aids in assessing familial oncological risks by focusing mostly on specific, highly penetrant cancer-susceptibility genes such as BRCA1/2, PTEN, or TP53 [11]. Therefore, no existing guidelines are suited for interpreting NGS data obtained from oncology patients assessed by general oncology practices.

Here, we report our first-hand experience with NGS analysis of a large population of cancer patients. We present the statistics on identified genetic alterations and their interpretations, along with a detailed dissection of methodological obstacles faced in course of the identification of such incidental findings.

\section{Methods}

\section{Sample collection and sequencing}

Tumor samples were presented by FFPE tissue blocks from each patient. Tumor genomic DNA was extracted from 4 to 8 freshly cut sections of FFPE tissue using
GeneRead DNA FFPE kit (Qiagen) according to the manufacturer's protocol, including the step of specific removal of deaminated cytosine residues by the enzyme Uracil-N-Glycosylase (UNG). The concentration of the DNA was determined using the Qubit dsDNA HS Assay Kit. DNA quality was evaluated by the PCR-based QuantumDNA kit (Evrogen).

Depending on the panels used, 409 or 411 genes were analysed. Target region amplification was performed employing two panels: Ion AmpliSeq Comprehensive Cancer Panel (Thermo Fisher Scientific Inc.) and the Atlas ABC panel. The Atlas ABC panel was designed via Ion Ampliseq Designer (Thermo Fisher Scientific Inc.) through the White Glove process and includes two primer pools, comprising 409 amplicons within 4 cancerrelated genes: BRCA1, BRCA2, ATM, and CHEK2. Ion AmpliSeq ${ }^{\mathrm{TM}}$ Comprehensive Cancer Panel (CCP) targeted 409 genes and 15,992 amplicons in four pools. Five nanograms of FFPE-derived (tumor) DNA were used to prepare sequencing libraries using the Ion Ampliseq library preparation kit v2.0 and The Ion Torrent Dual Barcode Kit 1-96 (Thermo Fisher Scientific Inc) according to the manufacturer's protocol. The quality and quantity of the barcoded libraries were determined using gel electrophoresis and Qubit 2.0 Fluorometer TM (Thermo Fisher Scientific Inc). Pooled libraries were combined and diluted to $10 \mathrm{pM}$ and templated on the Ion Chef and loaded onto an Ion 540 chip. The Ion 540 chip was sequenced on the Ion GeneStudio S5 System (Thermo Fisher Scientific).

\section{Data analysis and interpretation}

Raw sequence data analysis, including base calling and demultiplexing, was performed using the Torrent Suite Software v.4.0.2 (Thermo Fisher Scientific, Inc.) Sequenced reads were mapped using the human genome as a reference (version GRCh37.p13), employing Burrows-Wheeler Aligner (BWA-mem, version 0.7.7-r441) or software from the sequencing platform provider. Software from sequencing platform provider (Ion Torrent Variant Caller version 5.8-18) was used to call somatic SNVs and small InDels. Detected variants were classified as hotspot and non-hotspot based on prevalence in the COSMIC database (COSMIC count of 10 was used as the threshold) [12]. Filtering methods were different for candidate variations in positions of recurrent mutagenesis and all the others. Thresholds used for hotspot variant filtering were the following: coverage depth $>19$; number of mutant reads $>7$; variant allele frequency $>2 \%$. Thresholds used for non-hotspot variant filtering were the following: coverage depth $>19$; number of mutant reads $>9$; variant allele frequency $>5 \%$. Stand bias analysis was performed employing in-house scripts. Analysis of $\mathrm{CNVs}$ was performed using ONCOCNV 
software [13]. Minor-allele frequency data were referenced using the 1000 Genomes Project Database [14], the NHLBI GO Exome Sequencing Project [15], and the TOPMED project [16]. Further analysis was focused only on variants in genes potentially associated with the development of hereditary cancer syndromes: BRCA1, BRCA2, MLH1, MSH2, MSH6, PMS2, EPCAM, APC, MUTYH, CDKN2A, CDK4, TP53, PTEN, STK11, CDH1, BMPR1A, SMAD4, PALB2, CHEK2, ATM, NBN, BARD1, BRIP1, RAD51C, RAD51D, POLD1, POLE, GREM1, HOXB13, AXIN2, GALNT12, RPS20, RNF43, NTHL1, MSH3, SMARCB1, and BLM.

\section{Discrimination of variants on somatic and germline ISOWN}

The discrimination between somatic and likely-germline missense mutations was performed employing ISOWN [17] with further manual curation and manual tools. In summary, ISOWN is a machine-learning-based method designed to predict whether a certain variant is germline or somatic based on several factors, including population frequency, variant allele frequency (VAF), VAF of the adjacent polymorphisms, nucleotide composition, potential damaging effects, and presentation of a variant in databases. ISOWN classified each missense variant as germline or somatic. Since ISOWN is only intended for the discrimination of single nucleotide variants, small deletions and insertions were considered to be somatic or germline based solely on manual curation.

\section{Principles of manual curation}

To take into account additional factors that were not otherwise considered by ISOWN, manual curation was performed for all of the detected variants. While manually determining the origin of the detected variants, the following was considered: (1) VAF of passenger variants are generally lower than VAF of driver mutations [18]; (2) the likelihood of detecting pathogenic variants in genes potentially associated with the development of HCS (or other mendelian diseases) is lower in patients who do not have an overt hereditary disease according to the clinical diagnosis (including personal and family history of cancer, morphological and histological presentation of the disease); (3) if no CNVs are detected in locus, the chances of detecting a variant with VAF lower than tumor cellularity are low, and (4) the patterns of germline and somatic mutations in certain genes were taken into account [19-21]. As a result of manual curation, variants were classified as germline heterozygous, germline homozygous, somatic, or of uncertain origin.

\section{Variant interpretation}

Patient tumor samples were analyzed to identify germline variants, potential associations with hereditary cancer syndromes, as well as potential predictive and prognostic biomarkers. Clinical interpretation of detected variants was performed to identify their potential association with hereditary cancer syndromes and aimed at classifying variants as pathogenic, likely pathogenic, variants of uncertain significance (VUS), likely benign, or benign. Clinical significance of individual variants in BRCA1 and BRCA2 genes was estimated using the ENIGMA database [22], while variants in APC, EPCAM, MUTYH, CDH1, GALNT12, MSH2, MSH6, PMS2, and MLH1 genes were assessed according to the InSiGHT database [23]. For variants with no estimated pathogenicity in these genes according to the aforementioned databases, as well as variants in other genes, we conducted a literature search for predicted impact on protein function as well as case-control and functional studies, according to ACMG guidelines [24]. These variants were then classified based on SHERLOC guidelines [25]. Only variants classified by manual curation as germline heterozygous or uncertain were subjected to clinical interpretation.

\section{DNA Sanger sequencing}

Variants classified as uncertain by manual curation and pathogenic, likely pathogenic, or VUS based on clinical interpretation were validated by Sanger sequencing. Sanger sequencing was performed using the ABI PRISM BigDye Terminator Cycle Sequencing v.2.0 Ready Reaction kit and ABI PRISM 3730 DNA analyzer (Applied Biosystems) as previously described [26]. Blood samples were used for Sanger sequencing. All blood samples matched the corresponding tumor samples.

\section{Results}

Study population

From $07 / 2018$ to $12 / 2019,183$ unselected adult patients satisfying eligibility criteria (see methodology) were referred for comprehensive molecular profiling at the discretion of their oncologists. In all 183 tumors, collected from the 23 tumor sites, including 34 colorectal, 24 pancreatic, 18 lung, 16 ovarian, 15 breast, 11 stomach, and others, DNA was extracted and NGS was performed. According to Oncotree classification [27], these tumors belong to 67 different histological and molecular tumor types. All patients were profiled on the Comprehensive Cancer Panel (Ion Torrent), covering a coding sequence 
of 409 oncogenes and tumor-suppressor genes. For 132 patients, additional sequencing was performed to include comprehensive coverage of BRCA1/2, ATM, and CHEK2 genes (Atlas $\mathrm{ABC}$ panel). Additional Sanger sequencing was performed for 7 patients (3.8\%) with variants of likely germline origin and uncertain origin following manual validation to determine their somatic or germline origins The clinicopathological characteristics of the patients are shown in Table 1.

\section{Sequencing results and variant origin discrimination}

In total, from a sample of 183 patients, we detected 56 unique variants (Table 2, Fig. 1). Of those, 42 (75\%) were missense, $9(16 \%)$ were small insertions or deletions (indels), and 5 (9\%) were nonsense mutations.

Since the sequencing was performed in collected specimens only, the mutations found were classified as somatic, germline homozygous, germline heterozygous, or variants of uncertain origin based on machine learning algorithms (ISOWN) followed by manual validation or, for indel variants, based on manual validation only (see Methodology).

Overall, ISOWN predictions were concordant with the results of Sanger-based validation for the 41 (97\%) missense variants, including 10 germline and 31 somatic variants (Fig. 2). The most commonly mutated gene was TP53, which accounted for $48.2 \%$ of all the detected variants. All of the variants in TP53 were somatic, based on the results of both ISOWN and Sanger-based validation. Mutations in DDR genes (ATM, BLM, BRCA1, BRCA2, MLH1, MSH6, NBN, PMS2) accounted for up to $40 \%$ of the variants. The majority of observed variants were detected in patients with colorectal (35.7\% of all variants), gynecological (21.2\%), and pancreatic $(12.5 \%)$ cancers. A total of 38 variants across 32 patients were classified as somatic (Table 2, Fig. 2).

Following ACMG guidelines, clinical interpretation of germline variants or variants of uncertain origin was performed to classify them into pathogenic (PV), likely pathogenic (LPV), benign (BV), likely benign (LBV) variants, or variants of uncertain significance (VUS) [24]. In total, we detected 17 potentially-germline variants in 14 (8\%) patients with various tumor types. Genetic counseling was recommended for all patients with PV/LPV/ VUS variants of germline or uncertain origin. Germline variants classified as BV/LBV were not reported to the patients. Patients with the non-germline variants in genes associated with hereditary cancer syndrome (HCS) were not referred for genetic counseling. Five patients had both somatic and potentially-germline variants identified: 2 patients with colorectal, 1 patient with ovarian, 1 patient with stomach, and 1 patient with uterine cancers. In these patients, the somatic variants found were
Table 1 The clinicopathological characteristics of the patients

\begin{tabular}{|c|c|c|}
\hline Total & $\begin{array}{l}n \\
183\end{array}$ & $\%$ \\
\hline \multicolumn{3}{|l|}{ Age (years) at disease manifestation } \\
\hline Median (IQR) & \multicolumn{2}{|l|}{$56(44-65)$} \\
\hline$<40$ & 14 & 7.7 \\
\hline $40-49$ & 19 & 10.4 \\
\hline $50-59$ & 21 & 11.5 \\
\hline $60-69$ & 23 & 12.6 \\
\hline $70-79$ & 11 & 6.0 \\
\hline$\geq 80$ & 2 & 1.0 \\
\hline Unknown & 93 & 50.8 \\
\hline \multicolumn{3}{|l|}{ Sex } \\
\hline Male & 75 & 41.0 \\
\hline Female & 108 & 59.0 \\
\hline \multicolumn{3}{|l|}{ Tumor site } \\
\hline Colon and rectum & 34 & 18.6 \\
\hline Pancreatic & 24 & 13.1 \\
\hline Lung & 18 & 9.8 \\
\hline Ovary/fallopian tube & 16 & 8.7 \\
\hline Breast & 15 & 8.2 \\
\hline Stomach & 11 & 6.0 \\
\hline Cervix & 8 & 4.4 \\
\hline Other, including unknown primary & 8 & 4.4 \\
\hline Skin/melanoma & 8 & 4.4 \\
\hline Soft tissue & 7 & 3.8 \\
\hline Biliary & 6 & 3.3 \\
\hline Head and neck & 5 & 2.7 \\
\hline CNS/brain & 4 & 2.2 \\
\hline Bladder/urinary & 3 & 1.6 \\
\hline Uterus & 3 & 1.6 \\
\hline Kidney & 3 & 1.6 \\
\hline Bone & 2 & 1.1 \\
\hline Small bowel & 2 & 1.1 \\
\hline Prostate & 2 & 1.1 \\
\hline Ampulla of Vater & 1 & 0.5 \\
\hline Pleura & 1 & 0.5 \\
\hline Testis & 1 & 0.5 \\
\hline Liver & 1 & 0.5 \\
\hline \multicolumn{3}{|l|}{ Stage } \\
\hline I & 7 & 3.8 \\
\hline$\|$ & 15 & 8.2 \\
\hline III & 12 & 6.6 \\
\hline IV & 26 & 14.2 \\
\hline Not allowed to collect/not reported/unknown & 123 & 67.2 \\
\hline \multicolumn{3}{|l|}{ Metastasis stage } \\
\hline Mo & 65 & 35.5 \\
\hline M1 & 46 & 25.1 \\
\hline Not allowed to collect/not reported/MX/Unknown & 72 & 39.3 \\
\hline
\end{tabular}


Table 2 Results of mutation detection by gene

\begin{tabular}{|c|c|c|c|c|}
\hline \multirow[t]{2}{*}{ Gene } & \multirow[t]{2}{*}{ Total variants } & \multicolumn{2}{|c|}{ Origin status by manual curation } & \multirow{2}{*}{$\begin{array}{l}\text { PV(LPV) (+VUS) across } \\
\text { germline or uncertain } \\
\text { origin }\end{array}$} \\
\hline & & Somatic & $\begin{array}{l}\text { Germline (+variants of uncertain } \\
\text { origin) }\end{array}$ & \\
\hline TP53 & 27 & 27 & 0 & 0 \\
\hline APC & 5 & 3 & 2 & 2 \\
\hline ATM & 4 & 3 & 1 & 1 \\
\hline MSH6 & 3 & 0 & 3 & 3 \\
\hline PMS2 & 3 & 0 & 3 & 3 \\
\hline BRCA1 & 2 & 0 & 2 & 2 \\
\hline BRCA2 & 2 & 1 & 1 & $0(+1)$ \\
\hline CDKN2A & 2 & 1 & 1 & 0 \\
\hline BLM & 1 & 0 & 1 & 1 \\
\hline BMPR1A & 1 & 1 & 0 & 0 \\
\hline $\mathrm{CDH} 1$ & 1 & 0 & $0\left(+1^{*}\right)$ & 0 \\
\hline MLH1 & 1 & 0 & 1 & 1 \\
\hline MSH2 & 1 & 0 & 1 & 1 \\
\hline NBN & 1 & 1 & 0 & 0 \\
\hline SMAD4 & 1 & 1 & 0 & 0 \\
\hline SMARCB1 & 1 & 0 & $1^{*}$ & 0 \\
\hline Total & 56 & 38 & $17(+1)$ & $14(+1)$ \\
\hline
\end{tabular}

*Based on the results of manual validation. However, based on the results of Sanger sequencing on the patients' normal tissue, these variants were found to be somatic, and the patients were not referred for genetic counselling

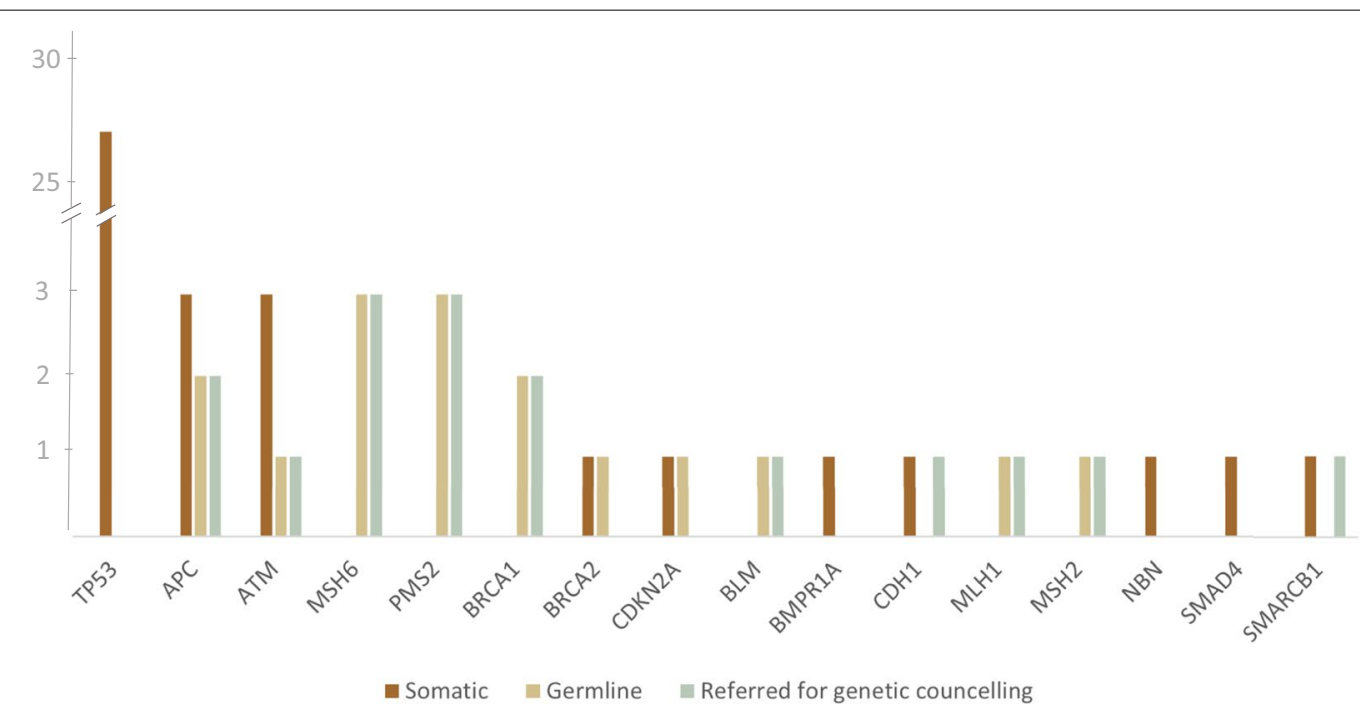

Fig. 1 The distribution of somatic and germline variants by gene. Variants were classified as germline or somatic based on the results of manual validation and Sanger sequencing. The number of patients referred for genetic counselling is also shown

accompanied by at least one potentially-germline PV or VUS.

Sanger sequencing validation was performed in 7 patients with suspected germline variants. Of those, five variants ( 1 in ATM, 1 in APC, 1 in BLM, 1 in BRCA2, 1 in MSH6) were found to be germline, and two-in
CDH1 and SMARCB2-were somatic. For the rest of the patients with PV/LPV/VUS variants of germline origin, Sanger sequencing was not performed due to one of the following reasons: (1) blood sample unavailable (2 cases); (2) patient preference (2 cases); (3) patient payor coverage circumstances (cost for Sanger sequencing was not 


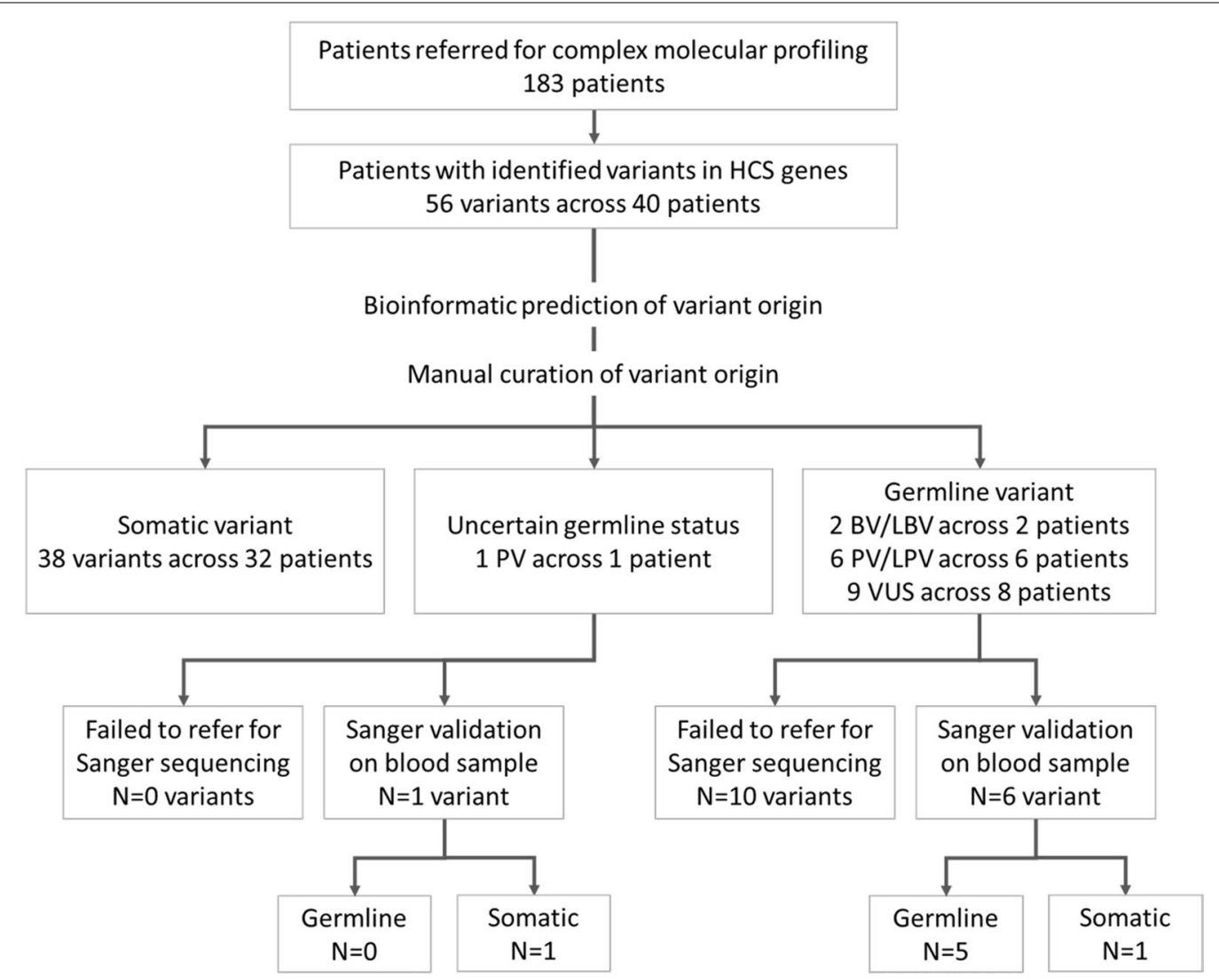

Fig. 2 Study design and major results of variant detection and validation. PV: pathogenic variant, LPV: likely pathogenic, BV: benign, LBV: likely benign, VUS: variant of uncertain significance

included in the cost for complex molecular profiling) (8 cases).

In two cases, Sanger sequencing failed to detect potentially-germline or variants of uncertain origin in patients' blood samples. In particular, variants in SMARCB1 (HGVSp ESNT000151345:p.R154L) and CDH1 (HGVSp ENST00000261769:p.Y302X) genes were predicted to be somatic by ISOWN. After a thorough assessment of patients' clinical characteristics and Sanger sequencing validation, these variants were labeled as likely germline (for SMARCB1) or of uncertain origin (for $\mathrm{CDH} 1$ ). In short, Sanger sequencing failed to detect these variants in the patients' normal tissue, thus justifying their somatic origin.

In one case, Sanger sequencing validation following ISOWN prediction allowed for the capture of the origin of a misclassified variant. Specifically, VUS in the ATM gene (ENST00000278616:p.S1584R, VAF 49.8\%) was detected in a patient with esophageal cancer and predicted to be somatic by ISOWN. Taking into account the clinical picture and the technical characteristics of the variant, we hypothesized that the variant may be, in fact, germline. Later, Sanger sequencing confirmed that the variant was germline, and the patient was referred for genetic counseling.

\section{Manual assessment of variant origin is beneficial over bioinformatics algorithms}

To assess the accuracy of tools for variant origin prediction, we manually assigned an origin (either germline, somatic or uncertain) to 1531 missense variants across 183 samples. Among them, 478 variants were found to be germline, 920 somatic, and 133 of uncertain origin (Fig. 3). Overall, ISOWN correctly predicted 436 (91\%) of the variants as germline and $742(80 \%)$ as somatic (Table 3). A subset of variants that were predicted to be somatic by ISOWN but classified as variants of uncertain origin based on manual validation had an average VAF of $48 \%$, which differs significantly from that of all variants manually classified as somatic $(28 \%$, p-value $<0.001)$, as well as from all variants predicted to be somatic by ISOWN (29\%, p-value <0.001) (Table 4). VAFs were not significantly different between the set of variants considered as somatic based on manual classification and 

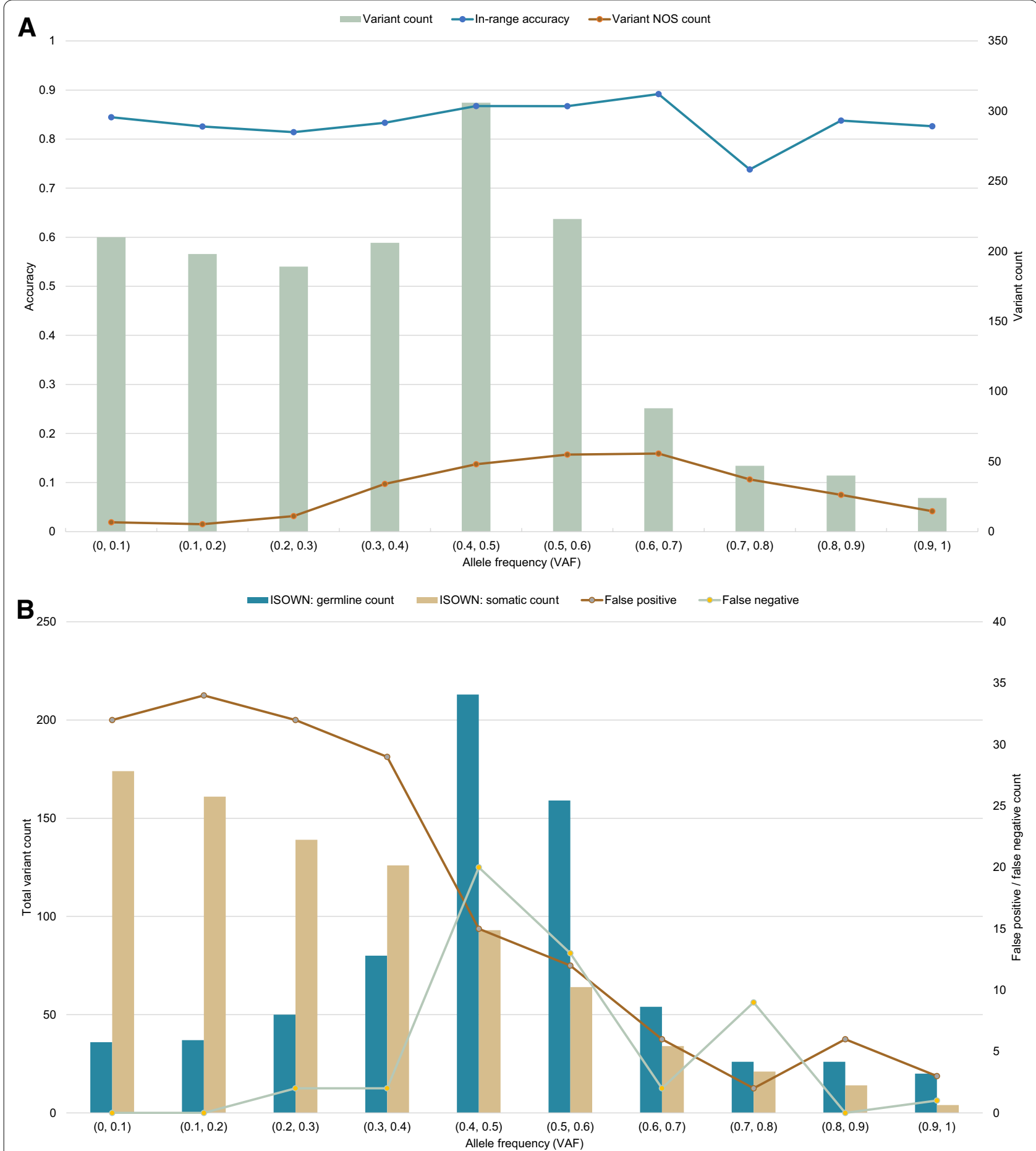

Fig. 3 Retrospective analysis of variant origin prediction results provided by bioinformatics software (ISOWN). Manual curation was used as the gold standard. ISOWN accuracy does not depend on the variant allele frequency (NOS — variants with uncertain origin, as considered by manual curation) (A), in contrast to false-positive and false-negative rates $(\mathbf{B})$. The same results were seen for different ranges of VAF distance between the studied variant and the known hotspot VAF in the same sample (C, D) 


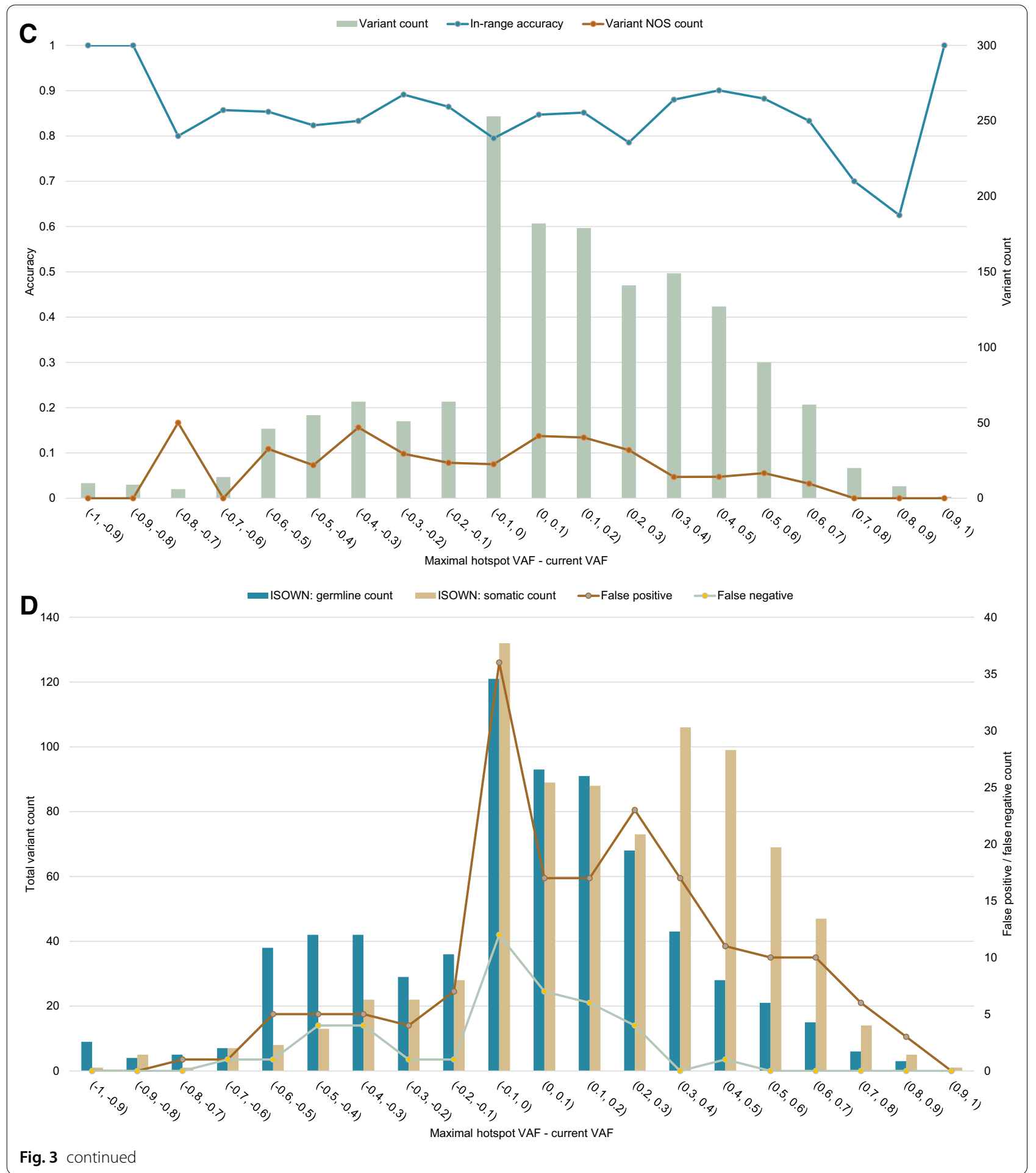

the set of variants predicted as being somatic based on ISOWN (mean $28 \%$ vs $29 \%$, p-value 0.14 ). Next, we compared the difference between maximal VAF of hotspot variants (defined as the maximum VAF across variants within a single molecular profile satisfying the following criteria: COSMIC count of 100 and more; population frequency based on data from the TOPMED project of 0.001 and less) and VAF of all of the studied variants. These VAFs were different in the subgroup of variants of uncertain origin that ISOWN predicted to be somatic 
Table 3 Accuracy of ISOWN predictions

\begin{tabular}{lll}
\hline Manual assignment & ISOWN & \\
\cline { 2 - 3 } & Germline & Somatic \\
\hline Germline & $\mathrm{TP}=436$ & $\mathrm{FN}=42$ \\
Somatic & $\mathrm{FP}=178$ & $\mathrm{TN}=742$ \\
Variant of uncertain origin & 87 & 46 \\
Accuracy of ISOWN prediction: $=84.26 \%$ & \\
Sensitivity: $=91.21 \%$ & & \\
Specificity: $=80.65 \%$ & & \\
Precision: $=71.01 \%$ & & \\
\hline
\end{tabular}

(mean-0.06\%), compared to all of the detected somatic variants $(21 \%, \mathrm{p}$-value $<0.001)$. This demonstrates how additional data, i.e. knowing the complete molecular profile of the patient, may be used in addition to the computationally predicted origin of individual variants.

ISOWN proved to be a useful tool for automated prediction of the variant origin; the overall sensitivity of ISOWN predictions was at $91.21 \%$; the overall accuracy was at $84.26 \%$; the precision or specificity was lower than that (Table 3). Notably, the accuracy of the predictions did not depend on variant allele frequency or the location of the variant concerning the hotspots (Fig. 2A, C). Across groups of variants with different VAFs, the accuracy was consistently higher than $70 \%$. A comparison of the ISOWN predictions to the results of manual assignment of variant origin shows that ISOWN had more false-positive results than false-negatives. It is clear that the false-positive results were prevalent at low VAFs, and false-negative results peaked around a VAF of $50 \%$ (Fig. 2B). False-negative results were consistently found to be located close to the hotspots and had an allele frequency close to $50 \%$. Other descriptors of false-negative variants did not significantly differ from descriptors of true somatic variants. A similar conclusion can be drawn for variants of uncertain significance that ISOWN classified as somatic (Table 4). Hence, our data suggest that ISOWN's predictions of somatic variants are least reliable for variants with $50 \%$ VAF located in the vicinity of known hotspots.

\section{The general problem of somatic/germline variant discrimination across other projects}

To estimate the risk of incorrect identification of the variant origin, somatic mutation data from the MSKIMPACT cancer molecular epidemiology project were analyzed [28]. Of 58,337 unique somatic mutations identified in the MSK-IMPACT, a total of 14,102 (24\%) were found in the dbSNP database (build 153). Of them, 1424 (2\% of the total unique somatic mutations) were found in the 1000 Genomes Project [14] and 7012 (12\% of the total unique somatic mutations) were found in the TOPMED project [16]. This indicates that between 12 and $24 \%$ of somatic variants may be located in the same genome positions as known germline variants. The frequency of germline variants may vary by ethnic background, making this an approximate estimation. Moreover, a total of $2188(4 \%)$ and 608 (1\%) somatic variants identified in the MSK-IMPACT project were previously annotated in the CLINVAR database as either pathogenic or likely pathogenic, respectively [29]. Of those, 45 variants were found in genes associated with HCS. These HSC variants were represented by a total of 82 occurrences across 78

Table 4 Variant annotation

\begin{tabular}{|c|c|c|c|c|c|c|c|}
\hline & \multicolumn{5}{|c|}{ Manual assignment } & \multicolumn{2}{|c|}{ ISOWN assignment } \\
\hline & \multirow[t]{2}{*}{ Germline } & \multirow[t]{2}{*}{ Somatic } & \multicolumn{3}{|c|}{ Variants of uncertain origin } & \multirow[t]{2}{*}{ Germline } & \multirow[t]{2}{*}{ Somatic } \\
\hline & & & Total & ISOWN: germline & ISOWN: somatic & & \\
\hline Total & 478 & 920 & 133 & 87 & 46 & 701 & 830 \\
\hline Annotated in COSMIC & $151(31.6 \%)$ & $329(35.8 \%)$ & $34(25.6 \%)$ & $20(23.0 \%)$ & $14(30.4 \%)$ & $204(29.1 \%)$ & $310(37.3 \%)$ \\
\hline Annotated in dbSNP & $423(88.5 \%)$ & $331(36.0 \%)$ & $82(61.7 \%)$ & $66(75.9 \%)$ & $16(34.8 \%)$ & $547(78.0 \%)$ & $289(34.8 \%)$ \\
\hline $\operatorname{VAF}($ mean $\pm S D)$ & $52 \% \pm 15 \%$ & $28 \% \pm 21 \%$ & $48 \% \pm 16 \%$ & $48 \% \pm 17 \%$ & $48 \% \pm 14 \%$ & $47 \% \pm 20 \%$ & $29 \% \pm 20 \%$ \\
\hline Minimal hotspot VAF (mean $\pm S D$ ) & $19 \% \pm 19 \%$ & $17 \% \pm 19 \%$ & $22 \% \pm 18 \%$ & $23 \% \pm 17 \%$ & $20 \% \pm 18 \%$ & $20 \% \pm 20 \%$ & $16 \% \pm 17 \%$ \\
\hline Maximal hotspot VAF (mean $\pm S D$ ) & $49 \% \pm 29 \%$ & $49 \% \pm 28 \%$ & $53 \% \pm 27 \%$ & $56 \% \pm 27 \%$ & $49 \% \pm 27 \%$ & $47 \% \pm 30 \%$ & $49 \% \pm 28 \%$ \\
\hline $\begin{array}{l}\text { Maximal hotspot VAF-current } \\
(\text { mean } \pm S D)\end{array}$ & $-7.6 \% \pm 32 \%$ & $21 \% \pm 31 \%$ & $5.2 \% \pm 28 \%$ & $8.0 \% \pm 28 \%$ & $-0.06 \% \pm 26 \%$ & $0.09 \% \pm 34 \%$ & $19 \% \pm 31 \%$ \\
\hline $\begin{array}{l}\text { Maximal hotspot VAF-minimal hotspot } \\
\text { VAF (mean } \pm \text { SD) }\end{array}$ & $26 \% \pm 25 \%$ & $31 \% \pm 25 \%$ & $31 \% \pm 25 \%$ & $33 \% \pm 25 \%$ & $28 \% \pm 25 \%$ & $27 \% \pm 26 \%$ & $32 \% \pm 25 \%$ \\
\hline EXAC frequency (mean) & $1.28 \%$ & $0.0017 \%$ & $0.004 \%$ & $0.005 \%$ & $0.002 \%$ & $0.88 \%$ & $0.0013 \%$ \\
\hline TOPMED frequency (mean) & $1.23 \%$ & $0.0012 \%$ & $0.0026 \%$ & $0.0029 \%$ & $0.002 \%$ & $0.84 \%$ & $0.0013 \%$ \\
\hline $1000 G$ frequency (mean) & $1.16 \%$ & $0.0015 \%$ & $0.0038 \%$ & $0.0037 \%$ & $0.0039 \%$ & $0.79 \%$ & $0.0014 \%$ \\
\hline
\end{tabular}


$(0.8 \%)$ different patients. For 11 ( $0.1 \%$ of patients, $95 \%$ CI $0.05-0.2 \%)$ of them, VAFs were in the range of 0.4 to 0.6 . Within the tumor, normal pairs assessed in the frame of the MSK-IMPACT project, this estimation does account for germline variants that could be detected. Nevertheless, our data indicate that automated variant origin discrimination may lead to an incorrect assessment in $1 \%$ of patients, and Sanger validation may be required. In other words, normal tissue specimen Sanger sequencing should be recommended in case PV/LPV or VUS variant is identified in any of the HCS genes.

Next, we analyzed a total of 32,10 , and 1 tumor molecular profiling reports generated by FoundationOne ${ }^{\circledR} \mathrm{CDx}$, FoundationOn ${ }^{\circledR} \mathrm{Heme}$, and FoundationOne ${ }^{\circledR}$ Liquid companion diagnostic tests, respectively (Foundation Medicine, Inc.). We found that out of 187 variants reported across 43 reports, 100 (53\%) were matched to an entry in the dbSNP database. In contrast, mutations annotated as somatic either by Sanger sequencing $(\mathrm{N}=920)$ or by ISOWN $(\mathrm{N}=830)$ were found in the dbSNP database with frequencies of only $36 \%$ and $35 \%$, respectively. Moreover, in the MSK-IMPACT samples, only $24 \%$ of somatic variants were annotated in dbSNP. This may indicate a bias towards reporting germline variants in tumor-only sequencing datasets. Across FMI reported variants, a total of $76(41 \%)$ and $32(17 \%)$ were present in TOPMed and 1000Genomes population databases, respectively. Furthermore, a total of 44 (24\%) variants had a population frequency of $0.1 \%$ and greater and a total of $10(5 \%)$ variants had a population frequency of $1 \%$ and greater. Such statistics point towards a germline origin of these variants rather than a somatic origin, while the high population frequency of these variants indicates a possible lack of relevance to carcinogenesis and indicates potential problems with reporting germline variants across tumor profiling providers.

\section{Discussion}

In oncology, NGS is predominantly used for the identification of somatic alterations. When found, these alterations guide therapeutic decisions on the applicability of the targeted therapies [30]. The majority of such alterations are either somatic mutations or fusions [28, 31]. Apart from somatic mutations, NGS is capable of identifying potentially germline variants, which may influence patient management as well as provide a rationale for timely genetic counseling and the implementation of screening the patients' relatives [32]. However, while performing the sequencing in tumor specimens only, one should rely on either computationally predicting whether a certain variant is a germline one, or resort to a secondary study of normal tissues by Sanger sequencing. Additionally, variant origin analysis may eliminate the reporting of a fraction of irrelevant variants, such as potentially benign, likely-germline variants, or commongenetic polymorphisms.

It is expected that the discrimination between somatic and germline mutations will remain a crucial problem for the molecular profiling providers who use only tumor specimens as samples. In our study, we describe realworld outcomes of performing this type of sequencing for cancer patients. We describe the main considerations for classifying variants as somatic or likely germline using ISOWN and Sanger sequence validation, as well as highlight the importance of Sanger sequencing.

Though ISOWN can accurately predict the origin of up to $99 \%$ of missense variants [17], manual curation was only performed for all the controversial missense and non-missense variants. Both ISOWN and manual interpretation have their limitations. As discussed earlier, ISOWN can only predict the origin of missense variants and cannot be used to annotate indels. Other limitations of ISOWN, as mentioned in the original article, include decreased accuracy in cancer types with lower mutational load [17], such as breast cancer. Moreover, we show that ISOWN is the least accurate in predicting the origin of variants with VAF of around $50 \%$ or VAFs located close to hotspots. Considering all the limitations of ISOWN, manual curation remains an essential part of variant interpretation. We show that a combination of ISOWN and manual curation is effective in assigning either somatic or germline origin to the variants observed in the clinical setting.

Since NGS is an imperfect means for the detection of germline variants and ISOWN might misclassify potentially-germline variants, we propose that adding a category of "variants of uncertain origin" may be useful in the framework of manual validation of the variants to denote the changes which may not be unequivocally classified as somatic or germline. This approach aids in avoiding the misclassification of the origin of certain potentiallygermline variants that otherwise would be classified as somatic. These variants should be further subjected to Sanger sequencing. In the case of the sequencing of the tumor samples, normal tissue Sanger sequencing may be required for at least $1 \%$ of cases referred for complex molecular profiling (95\% CI $0.62-0.97 \%$ ). In our real-world study, Sanger sequencing was required for 7 patients $(3.8 \%$, 95\% CI $1.5-7.7 \%)$ with pathogenic or likely pathogenic potentially-germline or variants of uncertain origin. When coupled with the results of the MSK-IMPACT project, our data demonstrate that collecting patients' normal tissue samples may be required for approximately $10 \%$ of real-world cases.

Apart from methodological obstacles in variant detection, the interpretation of sequencing variants as 
unequivocally germline or somatic remains challenging. Currently, several guidelines for the interpretation of the detected variants are available. The widely implemented ACMG guidelines [24], as well as their refined version, SHERLOCK [25], propose a 5-tier variant classification system. However, since these guidelines are focused on Mendelian hereditary conditions, their applicability to hereditary cancer syndromes is limited due to several reasons. First, patients with HCS may develop late-onset malignancies due to low expressivity of a trait [33], which precludes suspicion of its inherited nature. Secondly, the spectrum of malignancies among affected individuals in the same family may vary [34-37]. Monogenic hereditary cancer syndromes are rarely limited to the development of only one tumor type but rather associated with a range of malignancies. Coupled with variable penetrance and expressivity, both family history and variant segregation analysis may be used as strong or supporting evidence of pathogenicity or benign impact of a germline variant, as per ACMG guidelines, and complicate interpretation of the significance of a found variant.

As a consequence, an assessment of all variants in genes associated with HCS within the framework of tumoronly complex molecular profiling should be considered. Based on real-world results, we provide methodological guidance for this kind of research (Fig. 4). Even as bioinformatic tools aid in distinguishing somatic vs germline origin by assessing variant allele frequency, presence of a variant in databases, nucleotide composition, $\mathrm{CNV}$ analysis, and more [38], misclassification events may occur, thus, warranting manual curation. Here we show that the errors may persist even after manual curation. Such errors may lead to incorrect management of patient and family counselling. Therefore, after completion of manual curation, normal tissue sequencing validation is required for all variants identified as potentially germline (including variants of uncertain origin). Moreover, patient management may depend on the potential pathogenicity of variants identified as germline or potentially germline. The significance of VUS may further be clarified based on clinical data, like morphological characteristics of the disease, family history, or segregation of the variant in the family. Therefore, following genetic counseling, further assessment may be recommended to refer patients with PV/LPV germline or potentially-germline variants for Sanger validation and/or family segregation analysis. Finally, BV or LBV should not be reported, as per ACMG guidelines [24].

In terms of management and screening procedures for patients with PV or LPV germline variants, current NCCN guidelines on Genetic/Familial High-Risk Assessment $[11,39]$ provide information only for a handful of genes relevant to a limited amount of tumor types. The development of such guidelines is complicated by the uncertainty of case-control studies and a lack of consensus on the appropriate threshold of hazard ratio (HR) for the selection of patients for the screening, as well as the spectrum of relevant tumor types. Additionally, there are currently no available guidelines on the management of patients with PV in other highly penetrant genes, such as BAP1. Moreover, no guidelines discuss the management of patients harboring PV in genes who had already been affected by cancer and had no knowledge of the genetic basis for the disease before genetic testing.

With the rare exception of well-characterized missense variants, the majority of annotated PVs are either frameshift or nonsense mutations [29]. Most of the detected missense variants are classified as VUS, as many of them have not been previously studied and their effects on protein function remain unknown. For instance, for the BRCA 1 gene, only $4.5 \%$ of missense variants submitted to the ClinVar database are classified as PV, while 89\% are classified as VUS [21]. While in silico algorithms are useful for effect prediction, they may only provide supporting evidence for defining pathogenicity [40].

In our study, 7 patients harbored potentially germline VUS in genes associated with HCS, suggesting an underlying inherited nature of their tumor. Whether the patients' present diagnosis should be taken into account while interpreting potentially germline VUS should be further discussed by the scientific community. Efforts should be made to overcome methodological and clinical obstacles to the standardization of the genetic counseling of cancer patients referred for tumor molecular profiling.

The frequencies of incidental germline findings discovered during tumor molecular profiling were reported in many studies. In particular, Meric-Bernstam et al. showed that approximately $2.3 \%$ of patients with advanced cancer harbor previously unrecognised germline variants in genes associated with the development of HCS [41]. Some of these studies focus on specific tumor types. You et al. reported the overall frequency of pathogenic germline variants in patients with colorectal cancer of $9.9 \%$ [42]. In patients with lung cancer Tian et al. reported frequency of $\mathrm{PV} / \mathrm{LPV}$ as $3.8 \%$ [43]. Another study of patients with advanced cancer revealed the occurrence of germline PV in HRD genes as $17.8 \%$ [44].

The discrepancy of the reported frequencies might be explained by several factors, such as differences of study designs, patient populations, as well as selected tumor types. Moreover, overarching analysis is precluded by some studies reporting PV and LPV only, while some others including VUS. In our study, we report real world frequencies of incidental germline variants detected in the course of routine tumor molecular profiling. 


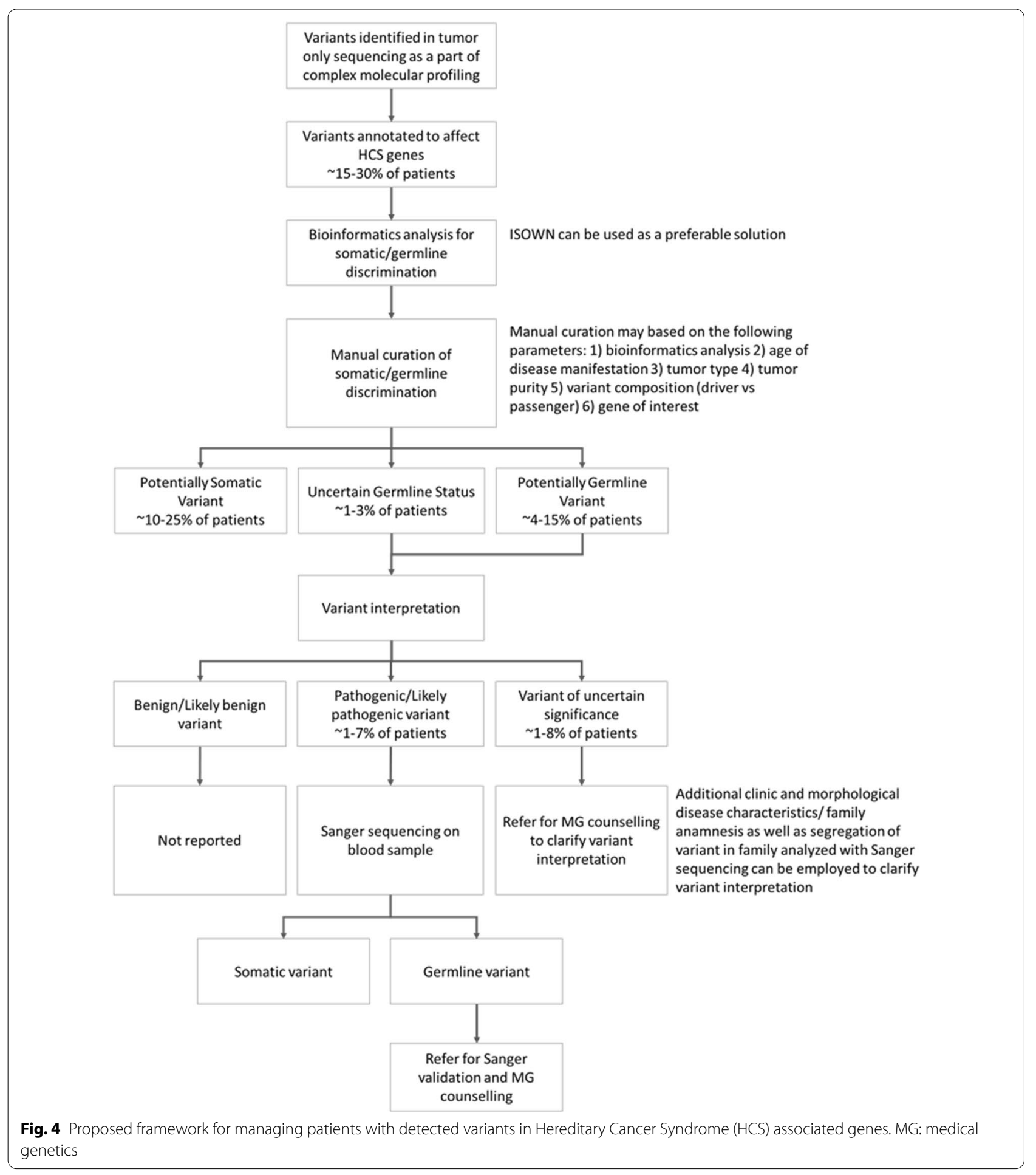

To sum up, in our study routine tumor molecular profiling revealed potentially-germline variants in $14(8 \%)$ patients with various tumor types referred for tumor molecular profiling. While the prediction of the variant origins may be done by computational tools, manual curation of the tumor-only sequencing results is paramount. We suggest adding an additional category of "variants of uncertain origin", which is of use when determining the origin of the sequencing variants. We highlight the importance of Sanger sequencing in patients' normal tissue for validation 
of the origin of PV/LPV/VUS variants that are either potentially germline, or of uncertain origin. We also discuss the obstacles for the interpretation of variants that are potentially germline or of uncertain significance in cancer patients referred to tumor molecular profiling.

\section{Conclusions}

Incidental findings of pathogenic germline variants are common in data from cancer patients referred for complex molecular profiling. We propose an algorithm for the management of variants in genes associated with HCS.

\section{Abbreviations \\ BV: Benign Variant; CNV: Copy Number Variation; FFPE: Formalin-Fixed Paraffin- Embedded; HCS: Hereditary Cancer Syndrome; LPV: Likely Pathogenic Variant; LBV: Likely Benign Variant; PV: Pathogenic Variant; SNV: Single-Nucleotide Vari- ant; VAF: Variant Allele Frequency; VUS: Variant of Unknown Significance.}

\section{Authors' contributions}

All authors have made significant contributions to the manuscript. All authors have read and approved the manuscript. All authors read and approved the final manuscript. Authors contributed as follows: AL — data analysis and interpretation, visualization, manuscript writing and editing; $Y S$ — data analysis, visualization, manuscript writing; DS—data collection; El, MS, AB —-manuscript revision and editing; ER —-methodology, manuscript editing; DV\&SM — manuscript writing and editing; TG—-methodology, manuscript writing; VM—study conceptualization; $\mathrm{Ml}$-study conceptualization and design.

\section{Funding}

The study was fully funded by Atlas Oncodiagnostic LLC.

\section{Availability of data and materials \\ Not applicable.}

\section{Declarations}

\section{Ethics approval and consent to participate}

The research was approved by the local ethics committee of Atlas Medical Center, LLC. The project was conducted following the principles expressed in the Declaration of Helsinki.

\section{Consent for publication}

Patients provided informed consent before participation in this retrospective study. All further analyses were based on the archival data that was stored in the database with no current connection to the patients'identifiers.

\section{Competing interests}

None to declare.

\section{Author details}

${ }^{1}$ Atlas Oncodiagnostics, LLC, Moscow, Russia. ${ }^{2}$ Sechenov University, Moscow, Russia. ${ }^{3}$ Salem Hospital, Salem, MA, USA. ${ }^{4}$ Department of chemotherapy №2, Federal State Budgetary Institution «N.N. Blokhin National Medical Research Center of Oncology» of the Ministry of Health of the Russian Federation, Moscow, Russia. ${ }^{5}$ Department of Oncogenetics, Institute of Higher and Additional Professional Education, Research Centre for Medical Genetics, Moscow, Russia. ${ }^{6}$ School of Systems Biology, George Mason University, Mannas, VA, USA ${ }^{7}$ Moscow Institute of Physics and Technology, Dolgoprudny, Moscow Region, Russia.

Received: 29 June 2021 Accepted: 5 January 2022

Published online: 15 January 2022

\section{References}

1. YU TM, Morrison C, Gold EJ, Tradonsky A, Layton AJ. Multiple biomarker testing tissue consumption and completion rates with single-gene tests and investigational use of oncomine Dx target test for advanced nonsmall-cell lung cancer: a single-center analysis. Clin Lung Caner. 2019. https://doi.org/10.1016/j.cllc.2018.08.010.

2. Takeyasu Y, Yoshida T, Motoi N, Teishikata T, Tanaka M, Matsumoto Y, et al. Feasibility of next-generation sequencing (Oncomine ${ }^{\mathrm{TM}}$ DX Target Test) for the screening of oncogenic mutations in advanced non-small-cell lung cancer patients. Jpn J Clin Oncol. 2021. https://doi.org/10.1093/jjco/ hyab059.

3. Kimura R, Ohtsuka T, Kubo M, Kajihara A, Fujii A, Watanabe Y, et al. FoundationOne ${ }^{\circledR} \mathrm{CDx}$ gene profiling in Japanese pancreatic ductal adenocarcinoma patients: a single-institution experience. Surg Today. 2020. https://doi.org/10.1007/s00595-020-02123-2.

4. Frampton GM, Fichtenholtz A, Otto GA, Wang K, Downing SR, He J, et al. Development and validation of a clinical cancer genomic profiling test based on massively parallel DNA sequencing. Nat Biotechnol. 2013. https://doi.org/10.1038/nbt.2696.

5. Ford L, Wolford JE, Brown SM, Randall LM. A profile on the FoundationFocus CDxBRCA tests. Expert Rev Mol Diagn. 2020. https://doi.org/10.1080/ 14737159.2020 .1701438$.

6. Arora S, Balasubramaniam S, Zhang H, Berman T, Narayan P, Suzman D, et al. FDA approval summary: olaparib monotherapy or in combination with bevacizumab for the maintenance treatment of patients with advanced ovarian cancer. Oncologist. 2021. https://doi.org/10.1002/onco. 13551.

7. Nagahashi M, Shimada Y, Ichikawa H, Kameyama H, Takabe K, Okuda S, et al. Next generation sequencing-based gene panel tests for the management of solid tumors. Cancer Sci. 2018. https://doi.org/10.1111/cas. 13837.

8. Yang Y, Muzny DM, Xia F, Niu Z, Person R, Ding Y, et al. Molecular findings among patients referred for clinical whole-exome sequencing. JAMA. 2014. https://doi.org/10.1001/jama.2014.14601.

9. Yushak ML, Han G, Bouberhan S, Epstein L, DiGiovanna MP, Mougalian SS, et al. Patient preferences regarding incidental genomic findings discovered during tumor profiling. Cancer. 2016. https://doi.org/10.1002/cncr. 29951.

10. Stjepanovic N, Stockley TL, Bedard PL, McCuaig JM, Aronson M, Holter S, et al. Additional germline findings from a tumor profiling program. BMC Med Genomics. 2018. https://doi.org/10.1186/s12920-018-0383-5.

11. Daly MB, Pal T, Berry MP, Buys SS, Dickson P, Domchek SM, et al. Genetic/ familial high-risk assessment: breast, ovarian, and pancreatic, Version 2.2021, NCCN clinical practice guidelines in oncology. J Natl Compr Canc Netw. 2021. https://doi.org/10.6004/jnccn.2021.0001.

12. Tate JG, Bamford S, Jubb HC, Sondka Z, Beare DM, Bindal N, et al. COSMIC: the catalogue of somatic mutations in cancer. Nucleic Acids Res. 2018. https://doi.org/10.1093/nar/gky1015.

13. Boeva V, Popova T, Lienard M, Toffoli S, Kamal M, Le Tourneau C, et al. Multi-factor data normalization enables the detection of copy number aberrations in amplicon sequencing data. Bioinformatics. 2014. https:// doi.org/10.1093/bioinformatics/btu436.

14. 1000 Genomes Project Consortium, Abecasis GR, Altshuler D, Auton A, Brooks LD, Durbin RM, et al. A map of human genome variation from population-scale sequencing. Nature. 2010. https://doi.org/10.1038/natur e09534

15. Fu W, O'Connor TD, Jun G, Kang HM, Abecasis G, Leal SM, et al. Analysis of 6,515 exomes reveals the recent origin of most human protein-coding variants. Nature. 2013. https://doi.org/10.1038/nature1 1690.

16. Kowalski MH, Qian H, Hou Z, Rosen JD, Tapia AL, Shan Y, et al. Use of $>100,000$ NHLBI Trans-Omics for Precision Medicine (TOPMed) Consortium whole genome sequences improves imputation quality and detection of rare variant associations in admixed African and Hispanic/ Latino populations. PLoS Genet. 2019. https://doi.org/10.1371/journal. pgen. 1008500 .

17. Kalatskaya I, Trinh QM, Spears M, McPherson JD, Bartlett JMS, Stein L. ISOWN: accurate somatic mutation identification in the absence of 
normal tissue controls. Genome Med. 2017. https://doi.org/10.1186/ s13073-017-0446-9.

18. Kumar S, Warrell J, Li S, McGillivray PD, Meyerson W, Salichos L, et al. Passenger mutations in more than 2,500 cancer genomes: overall molecular functional impact and consequences. Cell. 2020. https://doi.org/10. 1016/j.cell.2020.01.032.

19. Christie M, Jorissen RN, Mouradov D, Sakthianandeswaren A, Li S, Day F, et al. Different APC genotypes in proximal and distal sporadic colorectal cancers suggest distinct WNT/B-catenin signalling thresholds for tumourigenesis. Oncogene. 2013. https://doi.org/10.1038/onc.2012.486.

20. Mori Y, Nagse H, Ando H, Horii A, Ichii S, Nakatsuru S, et al. Somatic mutations of the APC gene in colorectal tumors: mutation cluster region in the APC gene. Hum Mol Genet. 1992. https://doi.org/10.1093/hmg/1.4.229.

21. Dines JN, Shirts BH, Slavin TP, Walsh T, King M-C, Fowler DM, et al. Systematic misclassification of missense variants in BRCA1 and BRCA2 "coldspots." Genet Med. 2020. https://doi.org/10.1038/s41436-019-0740-6.

22. Spurdle AB, Healey S, Devereau A, Hogervorst FBL, Monteiro ANA, Nathanson KL, et al. ENIGMA-evidence-based network for the interpretation of germline mutant alleles: an international initiative to evaluate risk and clinical significance associated with sequence variation in BRCA1 and BRCA2 genes. Hum Mutat. 2011. https://doi.org/10.1002/humu.21628.

23. Plazzer JP, Sijmons RH, Woods MO, Peltomäki P, Thompson B, Den Dunnen JT, et al. The InSiGHT database: utilizing 100 years of insights into Lynch Syndrome. Fam Cancer. 2013. https://doi.org/10.1007/ s10689-013-9616-0.

24. Richards S, Aziz N, Bale S, Bick D, Das S, et al. Standards and guidelines for the interpretation of sequence variants: a joint consensus recommendation of the American College of Medical Genetics and Genomics and the Association for Molecular Pathology. Genet Med. 2015. https://doi.org/10. 1038/gim.2015.30.

25. Nykamp K, Anderson M, Powers M, Garcia J, Herrera B, et al. Sherloc: a comprehensive refinement of the ACMG-AMP variant classification criteria. Genet Med. 2017. https://doi.org/10.1038/gim.2017.37.

26. Ivanov M, Matsvay A, Glazova O, Krasovskiy S, Usacheva M, Amelina $E$, et al. Targeted sequencing reveals complex, phenotype-correlated genotypes in cystic fibrosis. BMC Med Genomics. 2018. https://doi.org/ 10.1186/s12920-018-0328-z.

27. Kundra R, Zhang H, Sheridan R, Sirintrapun SJ, Wang A, Ochoa A, et al. OncoTree: a cancer classification system for precision oncology. JCO Clin Cancer Inform. 2021. https://doi.org/10.1200/CCl.20.00108.

28. Zehir A, Benayed R, Shah RH, Syed A, Middha S, Kim HR, et al. Mutational landscape of metastatic cancer revealed from prospective clinical sequencing of 10,000 patients. Nat Med. 2017. https://doi.org/10.1038/ nm.4333.

29. Landrum MJ, Lee JM, Riley GR, Jang W, Rubinstein WS, Church DM, et al. ClinVar: public archive of relationships among sequence variation and human phenotype. Nucl Acids Res. 2013. https://doi.org/10.1093/nar/ gkt1113.

30. Guan Y-F, Li G-R, Wang R-J, Yi Y-T, Yang L, Jiang D, et al. Application of nextgeneration sequencing in clinical oncology to advance personalized treatment of cancer. Chin J Cancer. 2012. https://doi.org/10.5732/cjc.012. 10216.

31. Cheng DT, Mitchell TN, Zehir A, Shah RH, Benayed R, Syed A, et al. Memorial sloan kettering-integrated mutation profiling of actionable cancer targets (MSK-IMPACT). J Mol Diagn. 2015. https://doi.org/10.1016/j. jmoldx.2014.12.006.

32. Stanislaw $C, X u e Y$, Wilcox WR. Genetic evaluation and testing for hereditary forms of cancer in the era of next-generation sequencing. Cancer Biol Med. 2016. https://doi.org/10.28092/j.issn.2095-3941.2016.0002.

33. Taeubner J, Wieczorek D, Yasin L, Brozou T, Borkhardt A, Kuhlen M. Penetrance and expressivity in inherited cancer predisposing syndromes. Trends Cancer. 2018. https://doi.org/10.1016/j.trecan.2018.09.002.

34. Wang J, Singh P, Yin K, Zhou J, Bao Y, Wu M, et al. Disease spectrum of breast cancer susceptibility genes. Front Oncol. 2021. https://doi.org/10. 3389/fonc. 2021.663419.

35. Mavaddat N, Peock S, Frost D, Ellis S, Platte R, Fineberg E, et al. Cancer risks for BRCA1 and BRCA2 mutation carriers: results from prospective analysis of EMBRACE. J Natl Cancer Inst. 2013. https://doi.org/10.1093/jnci/djt095.

36. Win AK, Lindor NM, Winship I, Tucker KM, Buchanan DD, Young JP, et al. Risks of colorectal and other cancers after endometrial cancer for women with lynch syndrome. J Natl Cancer Inst. 2013. https://doi.org/10.1093/ jnci/djs525.

37. Xicola RM, Li S, Rodriguez N, Reinecke P, Karam R, Speare V, et al. Clinical features and cancer risk in families with pathogenic $\mathrm{CDH} 1$ variants irrespective of clinical criteria. J Med Genet. 2019. https://doi.org/10.1136/ jmedgenet-2019-105991.

38. Sun JX, He Y, Sanford E, Montesion M, Frampton GM, Vignot S, et al. A computational approach to distinguish somatic vs. germline origin of genomic alterations from deep sequencing of cancer specimens without a matched normal. PLoS Comput Biol. 2018. https://doi.org/10.1371/ journal.pcbi.1005965.

39. Gupta S, Weiss JM, Axell L, Burke CA, Chen L, Chung DC, et al. Genetic/ Familial High-Risk Assessment: Colorectal. Version 1.2021, NCCN Clinical Practice Guidelines in Oncology. https://www.nccn.org/professionals/ physician_gls/pdf/genetics_colon.pdf. Accessed 24.06.2021

40. Kamps R, Brandão R, Bosch B, Paulussen A, Xanthoulea S, Blok M, et al. Next-generation sequencing in oncology: genetic diagnosis, risk prediction and cancer classification. Int J Mol Sci. 2017. https://doi.org/10.3390/ ijms180203.

41. Meric-Bernstam F, Brusco L, Daniels M, Wathoo C, Bailey AM, Strong L, et al. Incidental germline variants in 1000 advanced cancers on a prospective somatic genomic profiling protocol. Ann Oncol. 2016. https:// doi.org/10.1093/annonc/mdw018.

42. You YN, Borras E, Chang K, Price BA, Mork M, Chang GJ, et al. Detection of pathogenic germline variants among advanced colorectal cancer patients undergoing tumor genomic profiling for precision medicine. Dis Colon Rectum. 2020. https://doi.org/10.1097/DCR.0000000000001322.

43. Tian P, Cheng X, Zhao Z, Zhang Y, Bao C, Wang Y, et al. Spectrum of pathogenic germline mutations in Chinese lung cancer patients through next-generation sequencing. Pathol Oncol Res. 2020. https://doi.org/10. 1007/s12253-019-00771-5.

44. Bertelsen B, Tuxen IV, Yde CW, Gabrielaite M, Torp MH, Kinalis S, et al. High frequency of pathogenic germline variants within homologous recombination repair in patients with advanced cancer. NPJ Genom Med. 2019. https://doi.org/10.1038/s41525-019-0087-6.

\section{Publisher's Note}

Springer Nature remains neutral with regard to jurisdictional claims in published maps and institutional affiliations.

Ready to submit your research? Choose BMC and benefit from

- fast, convenient online submission

- thorough peer review by experienced researchers in your field

- rapid publication on acceptance

- support for research data, including large and complex data types

- gold Open Access which fosters wider collaboration and increased citations

- maximum visibility for your research: over $100 \mathrm{M}$ website views per year

At BMC, research is always in progress.

Learn more biomedcentral.com/submissions 